\title{
Optimal Inspection and Replacement Decisions for Multiple Failure Modes
}

\author{
Maarten-Jan Kallen* and Jan M. van Noortwijk \\ Delft University of Technology, Delft, Netherlands, and \\ HKV Consultants, Lelystad, Netherlands
}

\begin{abstract}
Cost functions, associated with the gamma process for modelling deterioration, are extended to include multiple failure modes. The application of these cost functions is demonstrated by an example in the process industry. We consider an elbow in a pipeline, which is susceptible to thinning due to corrosion and stress corrosion cracking. The optimal inspection interval and replacement levels are determined graphically and the effect of data (un)availability on the result is discussed.
\end{abstract}

\section{Introduction}

When modelling deterioration, engineers often deal with components or structures which can fail due to more than a single damage mechanism. In many cases, the engineer will choose to consider only one mechanism, which he deems to be most critical to the object under analysis. When there is little uncertainty involved, this assumption will be quite reasonable. In the case when there is high uncertainty involved with other possible damage mechanisms, it is advisable to incorporate these into the model as they can affect the confidence in the final decision.

In this paper, we will present one possible way of accomplishing this goal and we will present an example application in the process industry based on real-life data. In the example, we determine the cost-optimal combination of inspection interval and replacement condition level. The deterioration model, based on the gamma stochastic process, is presented together with the cost functions for the expected average costs per year over an unbounded horizon. Also, the measurements obtained during inspections are considered to be imperfect. Details on this approach can be found in [1], which is the predecessor of this paper.

Due to the use of a stochastic process to model the deterioration and renewal theory to account for costs of different actions and events, the methodology presents an interesting alternative to classic approaches to risk-based inspection (RBI) models, most of which use a first order reliability method (FORM) approach.

*Postal address: P.O.Box 2120, 8203 AC Lelystad, Netherlands. Email: m.j.kallen@hkv.nl 


\section{Deterioration and cost model}

For modelling the deterioration, we use the gamma stochastic process $\{X(t), t \geq 0\}$ with shape function $\alpha(t)>0$ and scale parameter $\beta>0$. The cumulative distribution of the gamma process at time $t$ is given by the cumulative gamma distribution:

$$
F_{X(t)}(x)=\operatorname{Pr}\{X(t) \leq x\}=\int_{\theta=0}^{x} \frac{\beta^{\alpha(t)}}{\Gamma(\alpha(t))} \theta^{\alpha(t)-1} e^{-\beta \theta} d \theta=\frac{\gamma(\alpha(t), \beta x)}{\Gamma(\alpha(t))},
$$

for $x \geq 0$. The quantity $P(a, x)=\gamma(a, x) / \Gamma(a)$ for $a>0$ and $x \geq 0$ is known as the incomplete gamma function.

Considering only a non-randomized inspection policy, the time interval between two consecutive inspections is $\Delta k$ years and inspections are performed at times $j \Delta k$ $(j=1,2,3, \ldots)$. The simplified notation $X_{j} \equiv X(j \Delta k)$ is used to represent the amount of deterioration at time $j \Delta k$.

\subsection{Probability of replacement and failure}

Using $r$ and $s$, with $0<r<s$, to represent the replacement and failure conditions respectively, we can determine the probability of no replacement after an inspection at time $(j-1) \Delta k$ and a failure at time $j \Delta k$ :

$$
\begin{aligned}
\operatorname{Pr} & \left\{X_{j-1} \leq r, X_{j}>s\right\} \\
& =\operatorname{Pr}\left\{X_{j-1} \leq r, X_{j}-X_{j-1}>s-X_{j-1}\right\} \\
& \stackrel{(*)}{=} \int_{\theta=0}^{r} \int_{\phi=s-\theta}^{\infty} f_{X_{j-1}}(\theta) f_{X_{j}-X_{j-1}}(\phi) d \phi d \theta \\
& =F_{X_{j-1}}(r)-F_{X_{j}}(s)+\int_{\theta=r}^{s} f_{X_{j-1}}(\theta) F_{X_{j}-X_{j-1}}(s-\theta) d \theta .
\end{aligned}
$$

At the equality sign labelled with $(*)$, we have used the fact that the increments in the gamma process are independent. From result (2), we can see that

$$
\operatorname{Pr}\left\{X_{j-1} \leq r, X_{j}>r\right\}=F_{X_{j-1}}(r)-F_{X_{j}}(r),
$$

which represents the probability of $X(t)$ passing the replacement condition level during the inspection interval $(j-1, j)$. Using results (2) and (3), we can determine all the probabilities which are needed for calculating the expected costs. For example, the probability of a replacement and no failure at time $j \Delta k$, when no replacement was performed at time $(j-1) \Delta k$, is given by

$$
\begin{aligned}
\operatorname{Pr} & \left\{X_{j-1} \leq r, r<X_{j} \leq s\right\} \\
& =\operatorname{Pr}\left\{X_{j-1} \leq r, X_{j}>r\right\}-\operatorname{Pr}\left\{X_{j-1} \leq r, X_{j}>s\right\} \\
& =F_{X_{j}}(s)-F_{X_{j}}(r)-\int_{\theta=r}^{s} f_{X_{j-1}}(\theta) F_{X_{j}-X_{j-1}}(s-\theta) d \theta .
\end{aligned}
$$




\subsection{Expected average costs per year}

Using a renewal theory approach, we consider the time periods in between two renewals (due to replacement or failure) as cycles. The expected average costs per time unit are then given by the expected costs incurred during the cycle divided by the expected length of the cycle: $\mathbb{E}(C) / \mathbb{E}\left(C_{I}\right)$, see e.g. [2]. Only non-discounted costs are used in the following sections and in the example. The expected cycle costs are given by

$$
\begin{aligned}
\mathbb{E}(C)=\sum_{i=1}^{\infty} c_{i} p_{i}=\sum_{j=1}^{\infty}\left[\left(j c_{I}+c_{P}\right) \operatorname{Pr}\left\{X_{j-1} \leq r, r<X_{j} \leq s\right\}+\right. \\
\left.+\left[(j-1) c_{I}+c_{F}\right] \operatorname{Pr}\left\{X_{j-1} \leq r, X_{j}>s\right\}\right],
\end{aligned}
$$

where $c_{i}$ are the total costs incurred during cycle $i$ and $p_{i}$ is the probability of incurring these costs during this cycle. When a failure is observed immediately and not only during inspections, the expected cycle length is given by

$$
\begin{aligned}
\mathbb{E}\left(C_{I}\right)=\sum_{i=1}^{\infty} i p_{i} & =\sum_{j=1}^{\infty}\left[j \Delta k \operatorname{Pr}\left\{X_{j-1} \leq r, r<X_{j} \leq s\right\}+\right. \\
+ & \left.\sum_{n=(j-1) \Delta k+1}^{j \Delta k} n \operatorname{Pr}\left\{X_{j-1} \leq r, X(n-1) \leq s, X(n)>s\right\}\right] .
\end{aligned}
$$

These expectations are also discussed in [3].

\subsection{Extension to multiple failure modes}

In order to implement multiple failure modes, we need to extend the existing failure and replacement probabilities. Here we consider two failure modes, namely failure due to corrosion and/or failure due to stress corrosion cracking (SCC). Both degradation mechanisms are modelled by their respective stochastic processes, represented by $V(t)$ and $W(t)$, with $t \geq 0$. The probability of no replacement due to inspection at time $(j-1) \Delta k$ and a failure at time $j \Delta k$ is represented by

$$
\operatorname{Pr}\left\{V_{j-1} \leq r_{v} \text { and } W_{j-1} \leq r_{w}, V_{j}>s_{v} \text { or } W_{j}>s_{w}\right\} \text {. }
$$

Assuming that both processes are independent and using the property

$$
\begin{aligned}
\operatorname{Pr}\{(A \cap B) \cap(C \cup D)\}= & \\
& \operatorname{Pr}\{A \cap B \cap C\}+\operatorname{Pr}\{A \cap B \cap D\}-\operatorname{Pr}\{A \cap B \cap C \cap D\},
\end{aligned}
$$

we can rearrange the probability (5) as follows:

$$
\begin{gathered}
\left.\operatorname{Pr}\left\{\left(V_{j-1} \leq r_{v} \cap W_{j-1} \leq r_{w}\right) \cap\left(V_{j}>s_{v} \cup W_{j}>s_{w}\right]\right)\right\}= \\
=\operatorname{Pr}\left\{V_{j-1} \leq r_{v}, V_{j}>s_{v}\right\} \operatorname{Pr}\left\{W_{j-1} \leq r_{w}\right\} \ldots \\
+\operatorname{Pr}\left\{W_{j-1} \leq r_{w}, W_{j}>s_{w}\right\} \operatorname{Pr}\left\{V_{j-1} \leq r_{v}\right\} \ldots \\
\quad-\operatorname{Pr}\left\{V_{j-1} \leq r_{v}, V_{j}>s_{v}\right\} \operatorname{Pr}\left\{W_{j-1} \leq r_{w}, W_{j}>s_{w}\right\} .
\end{gathered}
$$


The individual probabilities in this result have been treated in the previous sections, which leaves us with a simple and easy to implement model.

\section{Example: a pressurized steel pipeline elbow}

The elbow sections in pressurized pipelines are known to be very sensitive to a number of failure mechanisms. The following example considers one such elbow from a refinery, which transports the highly flammable and toxic gas n-Pentane. It therefore is essential that the component is inspected and replaced at appropriate times to avoid critical failure. The carbon steel, which the part is made of, is subject to thinning and to stress corrosion cracking.

The expectation of a (non-stationary) gamma process is modelled by a power law for the degradation:

$$
\mathbb{E}(X(t))=\frac{\alpha(t)}{\beta}=\frac{c t^{b}}{\beta} \equiv a t^{b} .
$$

The physical models for thinning and stress corrosion cracking are used to determine $a$ and $b$ in this expectation. Together with a coefficient of variation (see [1]), this expectation determines the shape function $\alpha(t)$ and the scale parameter $\beta$ of the gamma process.

For thinning the following (linear) state function is used:

$$
g_{T H}(t)=\left(t h-\frac{P d}{2 S}\right)-C t,
$$

where $t h$ is the initial wall thickness, $P$ is the operating pressure, $d$ is the component diameter and $C$ is the average thinning rate. Failure is considered to occur when $g(t)<0$, therefore the elbow fails when $C t>t h-P d /(2 S)$.

Stress corrosion cracking is not the same as cracking due to fatigue. It results from a combination of corrosion and a constant pressure or load, rather than from cyclic loads or sudden shocks. The state function for SCC is determined using standard theory for linear elastic fracture mechanics, see e.g. [4]. Failure due to SCC, occurs when the stress intensity $K=Y \sigma \sqrt{\pi a(t)}$ at the crack tip exceeds the critical stress intensity factor $K_{I c}$, where $a(t)=A t^{n}$ describes the crack growth as a function of time, $\sigma$ is the sum of external and internal stresses on the construction material and $Y$ is a geometrical correction factor to account for the shape of the component. From this information, the state function for SCC can be written in terms of a critical crack length $a_{\text {crit }}$ :

$$
g_{S C C}(t)=\frac{1}{\pi}\left(\frac{K_{I c}}{Y \sigma}\right)^{2}-a(t)=a_{c r i t}-A t^{n} .
$$

Failure due to stress corrosion cracking therefore occurs when $A t^{n}>a_{\text {crit }}$. The exponent $n$ is usually smaller than 1 and depends on whether or not the material has been stress relieved by post-weld heat treatment. The pipeline elbow in this example was not stress relieved and $n=0.65$ was used in the model. 


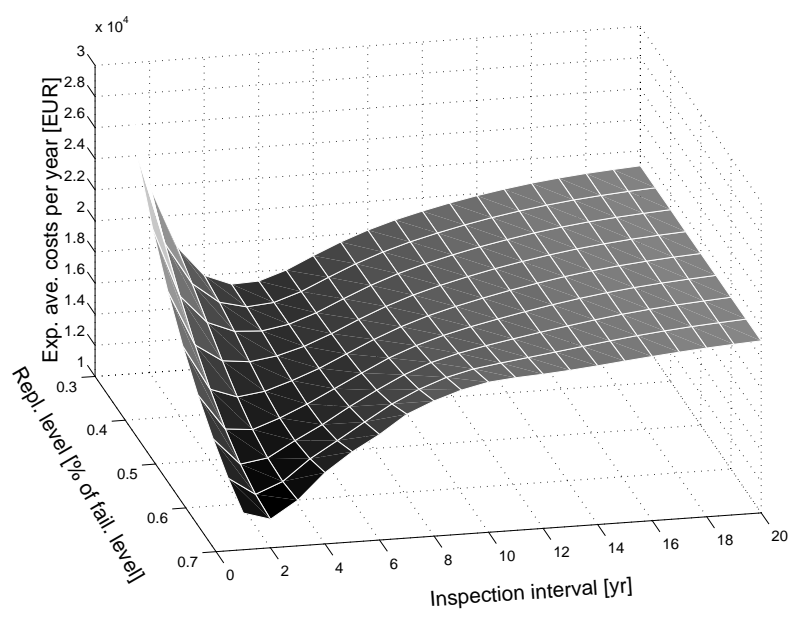

Figure 1: Expected average costs per year as a function of the inspection interval length $\Delta k$ and the percentage of the failure level at which the component is replaced.

Using these state functions and the probabilities and costs presented in the previous sections, the data of the pipeline elbow was put through the model to calculate the expected average costs per year. In order to improve the understanding of its behavior, the results are presented graphically in figure 1 as a function of the inspection interval and the replacement level. The replacement level is a percentage of the failure level, which is the same for both failure modes. For example, we can decide to replace the elbow when one or both of the processes have degraded to $50 \%$ of their respective failure conditions. The replacement percentage in figure 1 ranges from $30 \%$ to $70 \%$ in steps of $5 \%$ and the possible duration between inspections ranges from 1 to 20 years in steps of one year. In the figure, it can be clearly observed that a low replacement level will allow us to inspect less frequently, whereas a replacement level closer to the failure condition will require us to be careful and inspect more regularly. If we take the replacement level to be at $30 \%$, then the optimal inspection interval is about 5 to 6 years, whereas a replacement level at $70 \%$ of the failure condition will result in an optimum of 2 years.

If little or no data is available to the model, then the optimal inspection interval will be shorter compared to when more data is available. This effect is shown in figure 2, where the expected average costs per year are calculated with a replacement level at 50\%. It can be seen that the smaller uncertainty in the posterior density for the corrosion rate results in an optimal inspection interval of 5 years and lower expected costs. If the 5 wall thickness measurements would not have been available, then the prior density would be used and this results in an optimum at 3 years. Higher uncertainty in the rate of degradation will therefore result in shorter, more conservative, periods between consecutive inspections and higher expected costs. 


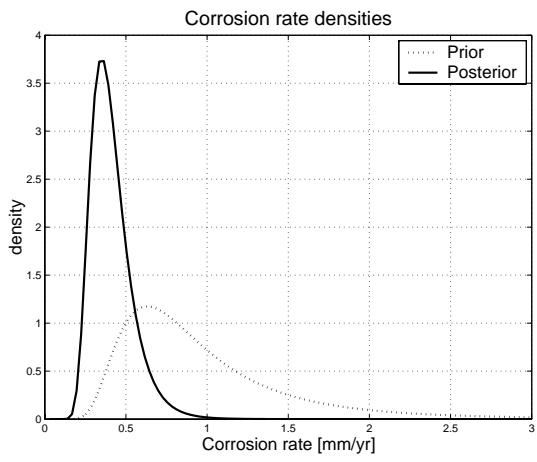

(a)

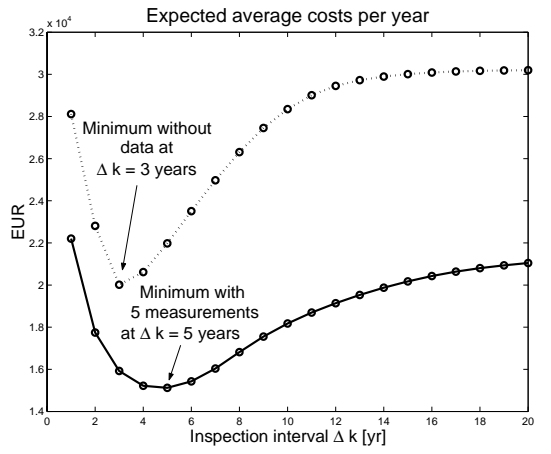

(b)

Figure 2: (a) The prior and posterior densities for the corrosion rate and (b) the effect of the (un)availability of data on the results for the expected average costs per year.

\section{Conclusions}

Experience with the model has shown that the damage mechanism with the shortest expected time to failure will largely determine the end results. The less critical process will not contribute significantly to the optimal decision unless its expected time to failure is close to that of the other process. In this case, the uncertainty of the second process will have a very strong effect on the end result. With this in mind, it is advisable to always incorporate all failure modes which are deemed to have an effect on the lifetime of the object under analysis. A simple and effective approach to implement multiple failure modes into the gamma process has been presented and demonstrated by a case study on an elbow in a steel pipeline at a refinery.

The author gratefully acknowledges the contribution of the pipeline elbow data by Det Norske Veritas in Rotterdam, the Netherlands.

\section{References}

[1] M. J. Kallen and J. M. van Noortwijk, "Inspection and maintenance decisions based on imperfect inspections," in Safety and Reliability - Proceedings of ESREL 2003: European Safety and Reliability Conference, June 15-18, Maastricht, the Netherlands, pp. 873-880, Swets \& Zeitlinger, 2003.

[2] S. M. Ross, Applied Probability Models with Optimization Applications. San Francisco: Holden-Day, Inc., 1970.

[3] J. M. van Noortwijk, M. Kok, and R. M. Cooke, "Optimal maintenance decisions for the sea-bed protection of the Eastern-Scheldt barrier," in Engineering Probabilistic Design and Maintenance for Flood Protection (R. M. Cooke, M. Mendel, and H. Vrijling, eds.), pp. 25-56, Kluwer Academic Publishers, 1997.

[4] D. Broek, Elementary Engineering Fracture Mechanics. Alphen aan de Rijn, The Netherlands: Sijthoff \& Noordhoff, 1978. 